\title{
Modeling, Simulation, and Testing of Surf Kites for Power Generation
}

\author{
Paul Williams, ${ }^{*}$ Bas Lansdorp,${ }^{\dagger}$ Richard Ruiterkamp, ${ }^{\ddagger}$ Wubbo Ockels ${ }^{\S}$ \\ Delft University of Technology, The Netherlands
}

\begin{abstract}
Non-powered flight vehicles such as kites can provide a means of transmitting wind energy from higher altitudes to the ground via tethers. At Delft University of Technology, construction and testing of such a high altitude wind machine is ongoing. The concept is called the Laddermill. It generates energy by pulling a line under high tension from a drum with a kite and retrieving it under low tension. The change in tension is achieved by changing the angle of attack and flight pattern of the kite. Although there is increased world wide interest for systems to extract wind energy from higher altitudes with kites, research into kite properties is limited. Such research however is required to enable the design of purpose-built kites for energy extraction. This paper presents testing of surf kites that was performed for model validation. Various models are discussed: a point model, a flexible multi-plate model and a rigid-body arc shaped model. Mathematical modeling and numerical simulations of the kite based on test data are presented.
\end{abstract}

\section{Introduction}

$\mathbf{I}^{\mathbf{r}}$ NTEREST in alternative energy sources has grown in the past decade due to the ever-increasing price of nonrenewable energy such as coal, gas, and oil. In recent years, there has been a concerted effort by governments around the world to increase the availability of renewable energy, resulting in regulations for the amount of renewable energy a country should produce. In the year $2000,1.2 \%$ of the consumption of Dutch electricity came from renewable sources [1]. In 2000, the Dutch government set new targets to comply with the Kyoto protocol, which aims to reduce the Dutch $\mathrm{CO}_{2}$ emissions. The broad target is to generate $5 \%$ of the electricity demand from renewable sources by 2010 , increasing to $10 \%$ by 2020 [1]. Among the sources that are considered "renewable" that can potentially be used for large-scale energy production are solar, hydroelectricity, wind, and biomass. Unfortunately, these technologies can only supply small amounts of energy per installed unit. The Laddermill $[2,3]$, a novel concept for extracting wind energy from high altitudes, aims to produce a quantum leap in "environmentally clean" electricity generation. The Laddermill concept has renewed interest in kite and tether dynamics.

The extraction of energy from the wind dates back hundreds of years, and is most commonly associated with windmills for pumping water or grinding grain by generating rotational motion from an array of blades. Typical windmills and wind turbines, beginning with the Hallady-Perry windmill design of the 1920s and 1930s, have been continually refined, altered, and developed over the decades in attempts to improve the efficiency of the designs. This has often only created small improvements in the system output. Many modern wind turbines are placed well above the ground at altitudes up to $120 \mathrm{~m}$ due to the fact that wind speeds increase dramatically with altitude. At altitude, factors such as turbulence, wind shear, and lack of other obstructions allows the wind to achieve much higher steady-state conditions than can be achieved at ground level.

In terms of an everyday usage and efficiency, ground-based wind turbine systems cannot be expected to generate a sufficient quantity of electricity relative to demand. One of the major problems with current technology for extracting energy from the wind is that long studies must be conducted to determine the optimal location for particular "wind farms" because of the extreme variability and intermittency of winds close to the Earth's surface. This, coupled with the cost of constructing wind farms and the low energy output from individual wind turbines leads to a relatively inefficient method for large-scale production of power. Another difficulty associated with the conventional wind farm is noise pollution, which limits the sizes of particular wind farms [4].

\footnotetext{
* Applied Researcher, AIAA Member, e-mail: tethered.systems@gmail.com.

${ }^{\dagger}$ PhD researcher, Faculty of Aerospace Engineering, E-mail: b.lansdorp@tudelft.nl.

* Applied Researcher, AIAA Member, e-mail: r.ruiterkamp@gmail.com

$\S$ ASSET Chairholder, Faculty of Aerospace Engineering, E-mail: w.j.ockels@tudelft.nl.
} 